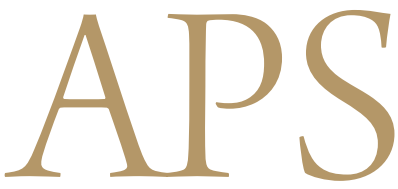

Archives of Plastic Surgery

\title{
Short-Term Strength Deficit Following Zone 1 Replantations
}

\author{
Si Young Roh ${ }^{1}$, Woo Cheol Shim ${ }^{1}$, Kyung Jin Lee ${ }^{1}$, Dong Chul Lee ${ }^{1}$, Jin Soo Kim ${ }^{1}$, \\ Jae-Won Yang ${ }^{2}$ \\ ${ }^{1}$ Department of Plastic and Reconstructive Surgery, Gwangmyeong Sungae Hospital, Gwangmyeong; ${ }^{2}$ Gangnam Jaejun Plastic Clinic for \\ Hand and Plastic Surgery, Pyeongtaek, Korea
}

Background Hand strength deficit following digital replantation is usually attributed to the mechanical deficiency of the replanted digit. Zone 1 replantation, however, should not be associated with any mechanical deficit, as the joint and tendon are intact. We evaluate shortterm motor functions in patients who have undergone single-digit zone 1 replantation.

Methods A single-institution retrospective review was performed for all patients who underwent zone 1 replantation. Hand and pinch strengths were evaluated using standard dynamometers. Each set of measurements was pooled according to follow-up periods (within 1 month, 1 to 2 months, 2 to 3 months, and after 3 months). The uninjured hand was used as reference for measurements.

Results The review identified 53 patients who had undergone zone 1 replantation and presented for follow-up visits. Compared to the uninjured hand, dynamometer measurements revealed significantly less strength for the hand with replanted digit at one month. The relative mean grip, pulp, and key pinch strength were $31 \%, 46 \%$, and $48 \%$ of the uninjured hand. These three strength measurements gradually increased, with relative strength measurements of 59\%, 70\%, and $78 \%$ for 4 -month follow up.

Conclusions Despite the lack of joint or tendon injury, strength of the injured hand was significantly lower than that of the uninjured hand during the 4 months following replantation. Improved rehabilitation strategies are needed to diminish the short-term negative impact that an isolated zone 1 replantation has on the overall hand strength.

Keywords: Hand strength / Pinch strength / Replantation / Range of motion, articular

\author{
Correspondence: Si Young Roh \\ Department of Plastic and \\ Reconstructive Surgery, \\ Gwangmyeong Sungae General \\ Hospital, 36 Digital-ro, Gwangmyeong \\ 14241, Korea \\ Tel: $+82-2-2680-7238$ \\ Fax: +82-2-2615-7218 \\ E-mail: psczero@gmail.com
}

Received: 17 Mar 2015 • Revised: 18 May $2015 \bullet$ Accepted: 1 Jun 2015

pISSN: 2234-6163 • elSSN: 2234-6171 • http://dx.doi.org/10.5999/aps.2015.42.5.614 • Arch Plast Surg 2015;42:614-618

\section{INTRODUCTION}

Strength and range of joint motion are the two most commonly evaluated functional outcomes following digital injury [1]. Total active motion (TAM) is the sum of active arc of flexion at the interphalangeal and metacarpophalangeal joints, and has been used as a good general metric for joint and tendon function [2].
Grip strength is another simple and standardized method of evaluating motor function. Measurement of grip strength is inexpensive and reliable, and has been widely used to evaluate nutritional status and hand function $[3,4]$.

Post-replantation functional outcomes are most commonly evaluated using TAM and grip strength. In a study comparing various replantation levels, Walaszek and Zyluk [5] reported 
that these functional outcomes were better for zone 1 replantations. Despite the lack of tendon injuries, zone 1 replantations result in hand functions that are significantly worse than those of the contralateral, non-injured hand [6]. Additionally, shortterm functional evaluations are not widely available for zone 1 injuries, with the implied assumption that such surviving digits are without any strength deficit. Hence, this study investigates short-term functional outcomes following Tamai [7] zone 1 replantation.

\section{METHODS}

A single-institution retrospective review was performed for all patients who had undergone zone 1 replantation between January 2011 and June 2014. The study excluded those patients who had multiple digit amputations, thumb amputations, bilateral hand injuries, and/or for reconstruction work in addition to the replantation. Demographic information, postoperative grip strength, and range of motion were collected.

The hand with replanted finger was kept in volar splint for 2 weeks, after which free finger movements were allowed. Physical therapy was started at 3 weeks. The physical therapist at our institution routinely measures hand strengths with a Jamar grip dynamometer (Patterson Medical, Bolingbrook, IL, USA) and a pinch dynamometers (Saehan Corp., Masan, Korea), using an established protocol [8]. Patients are seated with the shoulder adducted and neutrally rotated, elbow flexed at $90^{\circ}$ with the forearm in neutral position, and wrists between $0^{\circ}$ and $30^{\circ}$ of flexion and between $0^{\circ}$ and $15^{\circ}$ of ulnar deviation. Hand strength is evaluated in the order of grip, key pinch, and pulp pinch. The same dynamometer and pinch gauge is used for all participants. Each

\section{Table 1. Baseline characteristics for patients who have un-} dergone zone 1 replantation

\begin{tabular}{|lr|}
\hline Characteristic & $\mathbf{n}(\%)$ \\
\hline Sex & \\
Male & $36(68)$ \\
Female & $17(32)$ \\
Age (yr) & \\
$20-29$ & $6(11)$ \\
$30-39$ & $7(13)$ \\
$40-49$ & $22(42)$ \\
$50-70$ & $18(34)$ \\
Injured hand & \\
Right hand & $23(43)$ \\
Left hand & $30(57)$ \\
Injured finger & \\
Index finger & $17(32)$ \\
Middle finger & $14(26)$ \\
Ring finger & $11(21)$ \\
Little finger & $11(21)$ \\
\hline
\end{tabular}

examination is performed for three consecutive times at 1-minute intervals to reduce fatigue-induced measurement bias. Rangeof-motion is measured by the physical therapist at the same time [9].

Each postoperative measurement was used as an independent data set, and the measurements were analyzed in 4 groups according to the postoperative period (within 1 month, 1 to 2 months, 2 to 3 months, and after 3 months). Hand dominance was found to have no significant correlation with variations in grip strength measurements $[4,10]$, and hand strengths were compared to those measurements from contralateral hand. Strength measurements were expressed in lbs (\% of contralateral hand). All statistical analyses were performed using SPSS ver. 20.0 (IBM Corp., Armonk, NY, USA). We provided P-values using the independent two-samples $t$-test or one-way analysis of variance (ANOVA) test to signify outcome differences across the groups. Statistical significance was set at $\mathrm{P}<0.05$.

\section{RESULTS}

Out of the 322 patients with zone 1 amputation identified in the review, 41 patients were excluded for thumb replantations and 228 patients were excluded for multiple finger amputations or the need for additional reconstructive surgery. The remaining 53 patients consisted of 36 males and 17 females. The mean age was 45 years old (range, 20-70 years old). The mean duration of follow-up period was 9 weeks (range, 3-48 weeks). There were a total of 78 postoperative measurements ( 36 single visits, 9 double visits, and 8 triple visits) (Table 1$)$. Grip strength was significantly different between men $(37.22 \pm 18.50 \mathrm{lbs})$ and women $(\mathrm{M}=15.88 \pm 1.62 \mathrm{lbs})(\mathrm{P}<0.01)$. Patient age was not significantly related to grip strength $(\mathrm{P}=0.40)$.

The mean hand strength of the injured hand was all significantly lower, compared to the contralateral hand. Also, the mean range of motion for distal interphalangeal (DIP) joint was less for the

Table 2. Mean hand strengths and range of motion for all measurements

\begin{tabular}{|lccc|}
\hline Variable & $\begin{array}{c}\text { Injured } \\
\text { hand }\end{array}$ & $\begin{array}{c}\text { Contralateral } \\
\text { hand }\end{array}$ & $\%$ \\
\hline Hand function (lbs) & & & \\
$\quad$ Grip strength & $32.4 \pm 19.1$ & $68.4 \pm 24.4$ & 47 \\
Pulp pinch strength & $4.9 \pm 4.1$ & $9.9 \pm 3.1$ & 49 \\
$\quad$ Key pinch strength & $11.1 \pm 5.6$ & $17.5 \pm 4.6$ & 63 \\
Range of motion ( ${ }^{\circ}$ ) & & & \\
$\quad$ Metatarsophalangeal joint & $73 \pm 11$ & 90 & 81 \\
Proximal interphalangeal joint & $74 \pm 17$ & 110 & 67 \\
Distal interphalangeal joint & $25 \pm 17$ & 90 & 27 \\
\hline
\end{tabular}

The proportional strength measurement of the hand with replanted digit is represented as a percentage of the uninjured hand strength. 
Table 3. Average hand strengths and range of motion by monthly intervals

\begin{tabular}{|lcccc|}
\hline Variable & $\mathbf{1}$ mo & $\mathbf{2}$ mo & $\mathbf{3}$ mo & $\mathbf{4}$ mo \\
\hline Grip strength (normal hand) & $66.1 \pm 19.1$ & $66.9 \pm 24.0$ & $66.6 \pm 24.3$ & $74.6 \pm 28.9$ \\
Grip strength (injured hand) & $20.5 \pm 9.8$ & $31.9 \pm 20.3$ & $28.6 \pm 13.8$ & $43.7 \pm 20.1$ \\
Pulp pinch (normal hand) & $8.4 \pm 2.1$ & $10.3 \pm 3.6$ & $9.4 \pm 2.8$ & $10.2 \pm 2.3$ \\
Pulp pinch (injured hand) & $3.8 \pm 2.8$ & $4.7 \pm 4.4$ & $16.5 \pm 2.7$ & $7.1 \pm 4.5$ \\
Key pinch (normal hand) & $17.6 \pm 6.2$ & $17.4 \pm 4.5$ & $10.4 \pm 4.3$ & $18.6 \pm 3.7$ \\
Key pinch (injured hand) & $8.4 \pm 5.2$ & $10.5 \pm 5.8$ & $75 \pm 9$ & $14.5 \pm 5.3$ \\
Range of motion (MPJ) & $70 \pm 12$ & $71 \pm 12$ & $77 \pm 18$ & $78 \pm 6$ \\
Range of motion (PIPJ) & $66 \pm 17$ & $26 \pm 18$ & $32 \pm 12$ \\
Range of motion (DIPJ) & $27 \pm 22$ & $161 \pm 41$ & $30 \pm 17$ \\
Total active motion & $162 \pm 51$ & $173 \pm 39$ & $182 \pm 35$ \\
\hline As opposed to pinch strength measurements, grip strength is a global measure of the hand strength. Considering the fact that all of the patients had single-digit, zone 1 re- \\
plantation, this data suggests that immobilization of the whole hand may be a potential cause for significant decrease in hand function in the early recovery period. \\
Grip strength, pulp pinch, key pinch strength (lbs); range of motion (). & & \\
MPJ, metatarsophalangeal joint; PIPJ, proximal interphalangeal joint; DIPJ, distal interphalangeal joint. &
\end{tabular}

\section{Fig. 1. Hand strength for four months}

Horizontal axis indicates postoperative period. Vertical axis indicates strength in lbs.

\section{Grip strength, Key pinch, and Pulp pinch}

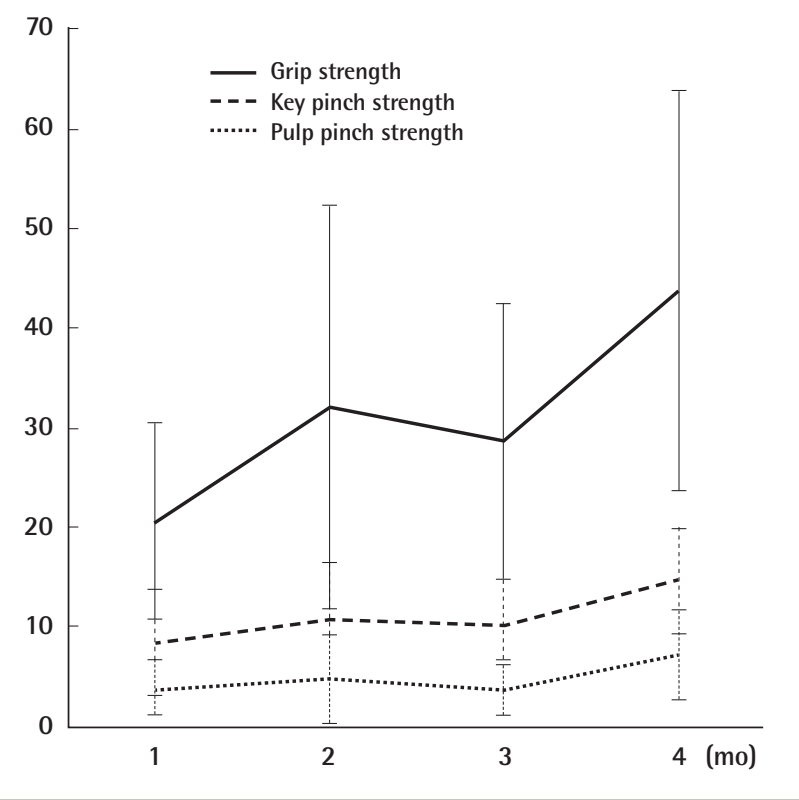

injured finger, compared to the counterpart joint in the uninjured hand (Table 2).

Compared to the uninjured hand, dynamometer measurements revealed significantly less strength for the hand with replanted digit at four weeks. The relative mean grip, pulp, and key pinch strength were $31 \%, 46 \%$, and $48 \%$ of the contralateral, uninjured hand. These three strength measurements gradually increased, with relative strength measurements of $59 \%, 70 \%$, and $78 \%$ for 4-month follow up (Table 3, Fig. 1). The difference in the strength measurements between early (within 1 month) and late (more

\section{Fig. 2. Total active motion for four months}

Horizontal axis indicates time after replantation, and vertical axis indicates total sum of joint motions.

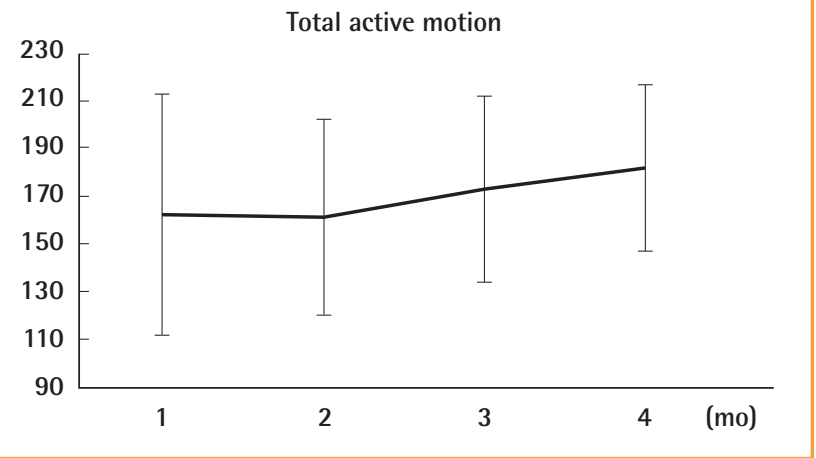

than 3 months) was statistically significant (one way ANOVA test, $\mathrm{F}=3.52, \mathrm{P}<0.05$ ).

The average TAM was $168^{\circ}$, which was $65 \%$ of the contralateral finger. Overall, TAM of the replanted digit improved over the 4-month period, from $162^{\circ}$ before 1 month to $181^{\circ}$ after 4 months. Whereas strength measurements improved without latency, initial recovery in range of motion was fairly slow for the second month at $161^{\circ}$ (Fig. 2).

\section{DISCUSSION}

Compared to more proximal amputations, zone 1 replantations are associated with shorter treatment duration and better functional outcomes because both the flexor tendon and DIP joint are intact. In a study comparing functional outcomes between replantation zones, Ross et al. [2] reported the total active range of motion to be $202^{\circ}$ for zone 1 vs. $126^{\circ}$ for zone 2 . In comparison, zone 4 replantations were associated with little to no motion at the DIP joint. Additionally, significant limitations in prox- 
imal interphalangeal joint motion have been observed when both profundus and superficialis tendons needed repair [11]. In a longterm follow up of 32 zone 1 replantations, Hattori et al. [12] found that the mean arc of DIP joint motion was $60^{\circ}$.

The grip strength of an injured hand is influenced by the number of replanted/revascularized digits and by the number of missing digits. Reported outcomes were better for single-digit replantations, especially in those patients with successfully replanted thumbs [5]. More specifically, the pinch and grip power of the replanted thumb has been reported to be $70 \%$ and $80 \%$ of the uninjured thumb, respectively [13]. Holmberg et al. [14] reported a mean total grip strength of $72 \%$ and a mean pinch grip strength of $69 \%$, compared to the uninjured hand. However, this study included a large number of incomplete and complete amputations. In our study of exclusively single-digit amputations, the mean grip strength of the injured hands was less than onethird of the uninjured hand at one month. This functional deterioration was observed in the early portion of recovery period (1 month). Our review of zone 1 replantation had precluded any patient with tendon injuries, yet the relative grip strength of $31 \%$ at one month suggested that patients were experiencing significant reduction in the ability to generate flexor force. The near $70 \%$ deficit in strength was unexpected and warrants a close investigation.

In previous studies, individual grip strength contributions have been evaluated for each finger. The middle finger is known to generate the most amount of power-about a third of grip strength. The ring and index fingers are symmetrical in force generation, with each contributing about $25 \%$ of the total grip strength. The little finger contributes approximately $15 \%[15,16]$.

Theoretically, strength deficits should be commensurate with the loss of individual strength of the finger that is unable to transfer the flexor force of the muscle. The worst-case scenario would be proximal amputation of the middle finger, which should result in a strength loss of around $30 \%$. However, our study has shown that distal zone 1 amputations without mechanical disruption of flexor mechanism can result in a relative grip strength deficit of $69 \%$ at 1 -month post operation.

In our review of the literature, we have found that most studies have focused on grip strength as surrogate markers for nutritional status and for long-term functional outcomes following hand operations [17]. While short-term grip strength following hand operations has not been studied extensively, this is a clinically relevant issue for those patients who return to a physically demanding work environment and accept this temporary but significant decline in hand strength as a natural consequence of a finger amputation.

This study has few limitations. Because of the retrospective study design, there may have been a selection bias towards patients with worse outcomes and for patients who wished to follow up more diligently or more frequently. However, we believe that the significant deterioration of grip strength is a real phenomenon and suspect that flexor weaknesses have resulted from the 2 weeks of splinting, by which the whole hand is immobilized to protect a single replanted digit. Pain may be a potential confounding factor.

In summary, grip strength was unexpectedly low in our group of patients who had undergone zone 1 replantation. With a recovery latency of about two months, patients experience significant limitations in generating maximal grip force for the first few months following a replantation. Further studies are necessary to evaluate whether this strength deficit is either due to a confounding variable such as pain or a clinical consequence of splinting the whole hand.

\section{REFERENCES}

1. Hume MC, Gellman H, McKellop H, et al. Functional range of motion of the joints of the hand. J Hand Surg Am 1990; 15:240-3.

2. Ross DC, Manktelow RT, Wells MT, et al. Tendon function after replantation: prognostic factors and strategies to enhance total active motion. Ann Plast Surg 2003;51:141-6.

3. Gunther CM, Burger A, Rickert M, et al. Grip strength in healthy caucasian adults: reference values. J Hand Surg Am 2008;33:558-65.

4. Shim JH, Roh SY, Kim JS, et al. Normative measurements of grip and pinch strengths of 21st century Korean population. Arch Plast Surg 2013;40:52-6.

5. Walaszek I, Zyluk A. Long term follow-up after finger replantation. J Hand Surg Eur Vol 2008;33:59-64.

6. Hasuo T, Nishi G, Tsuchiya D, et al. Fingertip replantations: importance of venous anastomosis and the clinical results. Hand Surg 2009; 14:1-6.

7. Tamai S. Twenty years' experience of limb replantation: review of 293 upper extremity replants. J Hand Surg Am 1982; 7:549-56.

8. Mathiowetz V, Kashman N, Volland G, et al. Grip and pinch strength: normative data for adults. Arch Phys Med Rehabil 1985;66:69-74.

9. Haidar SG, Kumar D, Bassi RS, et al. Average versus maximum grip strength: which is more consistent? J Hand Surg Br 2004;29:82-4.

10. Crosby CA, Wehbe MA, Mawr B. Hand strength: normative values.J Hand Surg Am 1994;19:665-70.

11. Waikakul S, Sakkarnkosol S, Vanadurongwan V, et al. Results 
of 1018 digital replantations in 552 patients. Injury 2000;31: 33-40.

12. Hattori Y, Doi K, Ikeda K, et al. Significance of venous anastomosis in fingertip replantation. Plast Reconstr Surg 2003; 111:1151-8.

13. Chen HC, Tang YB. Replantation of the thumb, especially avulsion. Hand Clin 2001;17:433-45.

14. Holmberg J, Lindgren B, Jutemark R. Replantation-revascularization and primary amputation in major hand injuries: resources spent on treatment and the indirect costs of sick leave in Sweden. J Hand Surg Br 1996;21:576-80.

15. Ohtsuki T. Inhibition of individual fingers during grip strength exertion. Ergonomics 1981;24:21-36.

16. MacDermid JC, Lee A, Richards RS, et al. Individual finger strength: are the ulnar digits "powerful"? J Hand Ther 2004; 17:364-7.

17. Norman K, Stobaus N, Gonzalez MC, et al. Hand grip strength: outcome predictor and marker of nutritional status. Clin Nutr 2011;30:135-42. 